\title{
The Efficacy of a Genetic Analysis of the BMPR2 Gene in a Patient with Severe Pulmonary Arterial Hypertension and an Atrial Septal Defect Treated with Bilateral Lung Transplantation
}

\begin{abstract}
Shunsuke Tatebe ${ }^{1}$, Koichiro Sugimura ${ }^{1}$, Tatsuo Aoki ${ }^{1}$, Saori Yamamoto ${ }^{1}$, Nobuhiro Yaoita ${ }^{1}$, Hideaki Suzuki ${ }^{1}$, Haruka Sato ${ }^{1}$, Katsuya Kozu ${ }^{1}$, Ryo Konno ${ }^{1}$, Kimio Satoh ${ }^{1}$, Koji Fukuda ${ }^{1}$, Osamu Adachi ${ }^{2}$, Ryoko Saito ${ }^{3}$, Norifumi Nakanishi ${ }^{4}$, Hiroko Morisaki ${ }^{5,6}$, Kotaro Oyama ${ }^{7}$, Yoshikatsu Saiki ${ }^{2}$, Yoshinori Okada ${ }^{8}$ and Hiroaki Shimokawa ${ }^{1}$
\end{abstract}

\begin{abstract}
:
Severe pulmonary arterial hypertension (PAH) rarely develops in children with an atrial septal defect (ASD), even those with a large defect. We herein report the case of a 27-year-old man with a moderate-sized secundum ASD and right ventricular failure due to severe PAH, which developed in his early teens. He was diagnosed as having a genetic mutation of the bone morphogenetic protein receptor-2 (BMPR2) gene and was successfully treated with bilateral lung transplantation with ASD path closure. In patients with congenital heart disease, a genetic analysis may provide information about the lifetime risk of developing PAH.
\end{abstract}

Key words: BMPR2 mutation, pulmonary arterial hypertension, atrial septal defect, Eisenmenger syndrome, lung transplantation, congenital heart disease

(Intern Med 56: 3193-3197, 2017)

(DOI: 10.2169/internalmedicine.8686-16)

\section{Introduction}

Severe pulmonary arterial hypertension (PAH) rarely occurs in children with atrial septal defect (ASD), even in patients with a large defect (1). This is because -in contrast to ventricular septal defect (VSD)-ASD only imposes a volume overload on the pulmonary circulation. Thus, pulmonary vascular disease (PVD) may not develop until the third or fourth decade of life. This observation raises the possibility that young ASD patients with severe PAH and patients with idiopathic PAH have common risk factors for developing PAH. A mutation in the bone morphogenetic protein receptor-2 (BMPR2) gene has been identified not only familial and idiopathic PAH but also in PAH associated with congenital heart disease (CHD) (2), indicating a common pathogenesis underlying the development of PAH in both groups.

We herein report the case of a 27-year-old man with a moderate-sized secundum ASD and right heart failure (RHF) due to severe $\mathrm{PAH}$, in whom a genetic analysis allowed us to understand the clinical course, including the childhood onset of PAH.

\section{Case Report}

A 27-year-old man with severe PAH and ASD was re-

\footnotetext{
${ }^{1}$ Department of Cardiovascular Medicine, Tohoku University Graduate School of Medicine, Japan, ${ }^{2}$ Department of Cardiovascular Surgery, Tohoku University Graduate School of Medicine, Japan, ${ }^{3}$ Department of Pathology, Tohoku University School of Medicine, Japan, ${ }^{4}$ Division of Pulmonary Circulation, Department of Cardiovascular Medicine, National Cerebral and Cardiovascular Center, Japan, ${ }^{5}$ Department of Bioscience and Genetics, National Cerebral and Cardiovascular Center Research Institute, Japan, ${ }^{6}$ Department of Medical Genetics, Sakakibara Heart Institute, Japan, ${ }^{7}$ Department of Pediatrics, Iwate Medical University, Japan and ${ }^{8}$ Department of Thoracic Surgery, Institute of Development, Aging and Cancer, Tohoku University, Japan

Received: December 9, 2016; Accepted: April 12, 2017; Advance Publication by J-STAGE: October 11, 2017

Correspondence to Dr. Shunsuke Tatabe, shuntatebe@ cardio.med.tohoku.ac.jp
} 
Table. Time-course of Hemodynamic Data.

\begin{tabular}{lrrrrr}
\hline & \multicolumn{2}{c}{13 years old } & \multicolumn{2}{c}{27 years old } & 30 years old \\
\cline { 2 - 6 } & Baseline & O2 (10L/min) & Baseline & NO (40ppm) & $\begin{array}{c}\text { (3 months after LTx }) \\
\text { Baseline }\end{array}$ \\
\hline PAP, mmHg & $102 / 49(69)$ & $102 / 43(66)$ & $60 / 35(48)$ & $62 / 37(48)$ & $22 / 8(13)$ \\
mRAP, mmHg & 6 & 6 & 7 & 4 & 0 \\
mPCWP, mmHg & 6 & 7 & 6 & 5 & 9 \\
AoP, mmHg & $104 / 78(87)$ & $102 / 71(84)$ & $95 / 78(80)$ & $105 / 73(80)$ & $143 / 80(100)$ \\
Qp, L/min & 3.75 & 3.11 & 2.06 & 2.13 & 4.64 \\
Qs, L/min & 3.38 & 2.72 & 2.46 & 2.62 & 4.64 \\
Qp/Qs & 1.05 & 1.15 & 0.84 & 0.81 & 1.0 \\
Rp, Wood Units & 16.8 & 18.6 & 20.4 & 20.2 & 0.9 \\
Rs, Wood Units & 23.9 & 28.7 & 29.7 & 29.0 & 21.6 \\
Rp/Rs & 0.74 & 0.65 & 0.69 & 0.70 & 0.04 \\
\hline AoP. & & &
\end{tabular}

AoP: aortic pressure, LTx: lung transplantation, mPCWP: mean pulmonary capillary wedge pressure, mRAP: mean right atrial pressure, PAP: pulmonary artery pressure, Qp: pulmonary blood flow, Qs: systemic blood flow, Qp/Qs: pulmonary to systemic flow ratio, Rp: pulmonary vascular resistance, Rs: systemic valcular resistance, Rp/Rs: pulmonary to systemic resistance ratio

ferred to Tohoku University Hospital to evaluate further treatment options in August 2012. Moderate cyanosis was initially noted at 13 years of age when he caught a cold. Right heart catheterization (RHC) at the referring hospital revealed severe PAH with irreversible PVD (Table) and a moderate-sized secundum ASD of $15 \mathrm{~mm}$ in size, with a bidirectional shunt. Because there was no indication for surgical ASD closure, treatment with beraprost sodium was started. However, his shortness of breath on exertion and general fatigue had gradually worsened over the past 5 years, despite the administration of bosentan and sildenafil. Syncope was also noted 2 years prior to his admission to our hospital. There was no family history of PAH or CHD.

On physical examination, his blood pressure was 106/64 $\mathrm{mmHg}$, and his pulse rate $90 \mathrm{bpm}$. Moderate cyanosis, an percutaneous oxygen saturation $\left(\mathrm{SpO}_{2}\right)$ of $80 \%$ in room air and clubbed fingers were observed. The precordial impulses were visible and palpable. Auscultation revealed an accentuated pulmonic component of S2, a grade 2/6 ejection systolic murmur along the lower left sternal border, and a rightsided S3 in the tricuspid region. Blood examination demonstrated secondary polycythemia with a hemoglobin level of $20.1 \mathrm{~g} / \mathrm{dL}$, a hematocrit value of $60 \%$, and elevated braintype natriuretic peptide (BNP) level of $499 \mathrm{pg} / \mathrm{mL}$. Chest Xray showed marked enlargement of the cardiac silhouette and dilatation of the hilar pulmonary arterial shadow (Fig. 1A). Furthermore, a 12-lead electrocardiogram (ECG) demonstrated a normal sinus rhythm, right axis deviation, first-degree atrioventricular block, and complete right bundle block with a QRS duration of $200 \mathrm{~ms}$ (Fig. 1B). Transthoracic echocardiography showed severely impaired systolic function of the right ventricle (RV) with a tricuspid annular plane systolic excursion of $11 \mathrm{~mm}$ and an RV fractional area change of $14 \%$. The RV was markedly enlarged (Fig. 1C). Tricuspid regurgitation was moderate with an estimated RV pressure of $75 \mathrm{mmHg}$. A secundum ASD of $16 \mathrm{~mm}$ in diameter with bidirectional flow was also identified (Fig. 1C).
The left ventricular ejection fraction (LVEF), as measured by cardiac magnetic resonance imaging decreased to $35 \%$ because of interventricular septal bowing and compression of the left ventricle (LV) (Fig. 1D). Chest CT showed no evidence of thromboembolism, tumors or lung disorders such as chronic obstructive or interstitial lung disease. Spirometry showed a vital capacity of $3.9 \mathrm{~L}$ (83\% of the predicted) and an forced expiratory volume (FEV) 1/forced vital capacity (FVC) ratio of $74 \%$. Although both the lung diffusion capacity of carbon monoxide (DLCO)(89.6\% of the predicted value) and the diffusing capacity divided by the alveolar volume (DLCO/VA)(89.6\% of the predicted value) were within the normal limits, the patient's exercise capacity was decreased; the 6-minute-walk distance was $378 \mathrm{~m}$, with a marked drop in $\mathrm{SpO}_{2}$ from $81 \%$ to $68 \%$ and a peak oxygen consumption of $11.3 \mathrm{~mL} / \mathrm{kg} / \mathrm{min}$ on bicycle ergometer. RHC revealed a pulmonary capillary wedge pressure of 6 $\mathrm{mmHg}$, mean pulmonary arterial pressure of $48 \mathrm{mmHg}$, mean RA pressure of $7 \mathrm{mmHg}$ (Table). The pulmonary to systemic flow ratio was 0.84 . The pulmonary and systemic vascular resistance was 20.4 and 29.7 Wood Units, respectively. No pulmonary vasoreactivity to inhaled nitric oxide (40 ppm for 10 minutes) was noted.

Given the poor prognosis of $\mathrm{PAH}$ with medically refractory RHF, the patient was referred for lung transplantation and was registered in the Japan Organ Transplantation Network in December 2012. A genetic analysis revealed a heterozygous missense mutation in exon 12 of the $B M P R 2$ gene (NM_001204.6: c.2474A>G), leading to the substitution of tryptophan to cysteine at amino acid position 825 (p.Tyr825Cys). Parental material was not available.

Three years later, the patient underwent bilateral lung transplantation and concomitant cardiac surgery. A secundum ASD of $16 \mathrm{~mm}$ was surgically confirmed and was closed with an autologous pericardial patch. A histological examination revealed increased muscularization of the intraacinar arterioles, medial hypertrophy and intimal thickening 

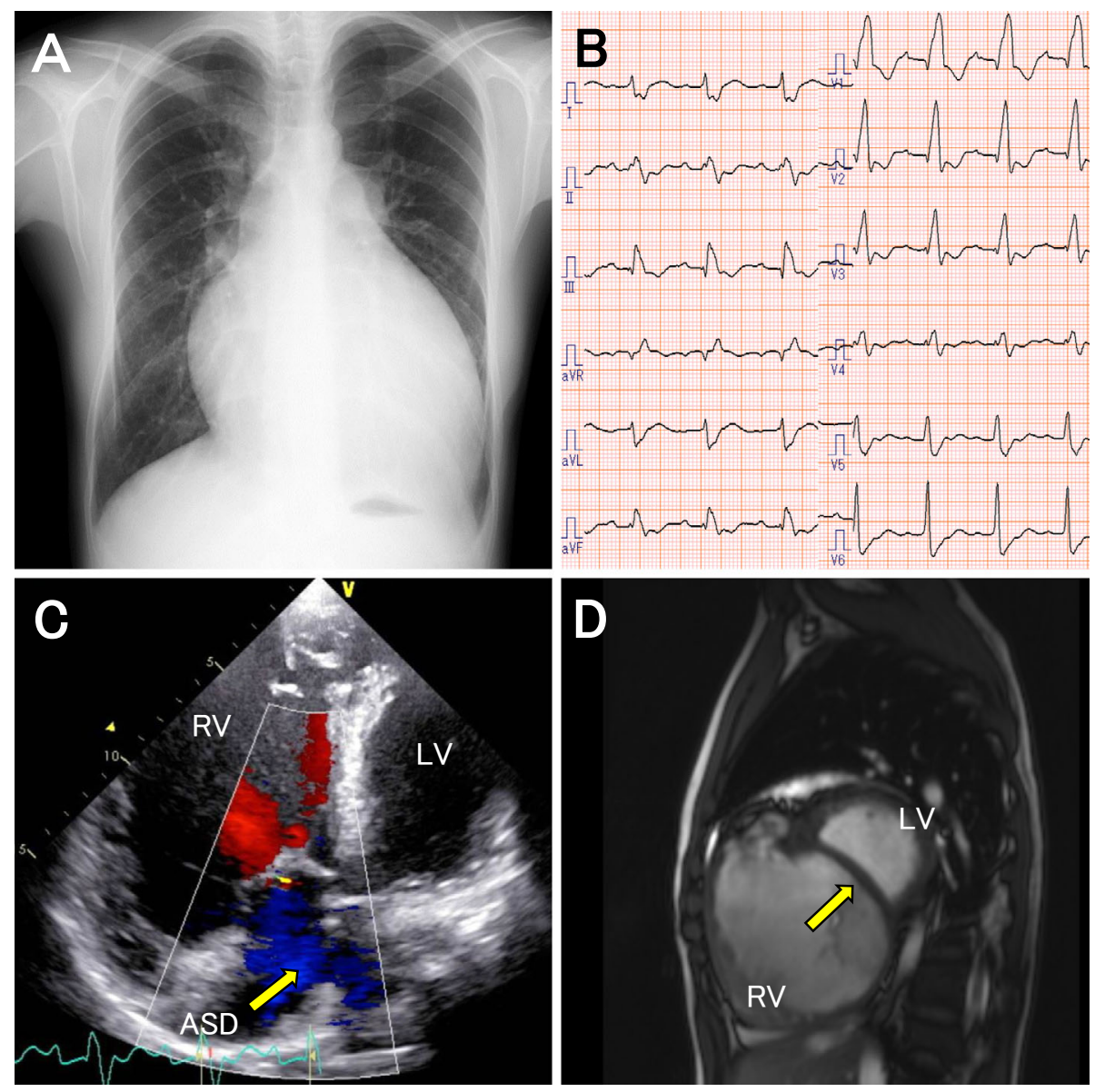

Figure 1. The examinations preformed on admission. (A) Marked enlargement of the heart silhouette on a chest X-ray. (B) Twelve-lead ECG showing first-degree atrioventricular block, right axis deviation, and complete right bundle branch block with a QRS duration of $200 \mathrm{~ms}$. (C) The apical 4-chamber view of the transthoracic echocardiogram with color flow Doppler demonstrating severe enlargement of the right ventricle and the secundum atrial septal defect of $16 \mathbf{~ m m}$ with a right to left flow (arrow). (D) A short-axis image obtained by cardiac MRI showing the massive enlargement of the right ventricle, leading to interventricular septal bowing toward the left ventricle (arrow).

of the small pulmonary arteries, and multiple channels at branch points of the large preacinar pulmonary arteries (Fig. 2A-C), the latter of which was thought to have developed as a result of plexiform lesions. The postoperative course was complicated by symptomatic ventricular tachycardia (VT), possibly due to preexisting myocardial damage. The patient's VT was successfully controlled by the combination of bisoprolol, mexiletine and sotalol. A subcutaneous implantable cardioverter-defibrillator (sICD) was implanted to allow the use of immunosuppressive drugs for preventing allograft rejection. At 6 months after the lung transplantation, the patient was discharged with a marked improvement in his cardiopulmonary symptoms. RHC before discharge confirmed the complete resolution of PAH with a normal cardiac output (Table). However, biventricular dysfunction partially remained with an LVEF of $52 \%$ and a right ventricular ejection fraction (RVEF) of $42 \%$ on cardiac MRI.

\section{Discussion}

PVD can occur in various types of CHD, leading to severe PAH and a fatal outcome (3). The causes of PVD associated with CHD are multifactorial and include chronic volume and pressure overload of the pulmonary artery due to left-to-right shunt, hypoxic vasoconstriction, and elevated pulmonary venous pressure (3). Although the time of the onset of advanced PVD varies in each patient according to their anatomy and physiology, an isolated ASD does not usually cause severe PAH in younger patients because the pulmonary vasculature is only exposed to excess pulmonary blood flow (4). In contrast, the vast majority of patients with unrestrictive VSD will develop severe PVD if they are not treated within the first years of life because the pulmonary vasculature is exposed to both an increased pulmonary flow and increased systemic arterial pressure (4). The present case was interesting because the patient had severe PAH with irreversible PVD at his initial presentation at 13 years 

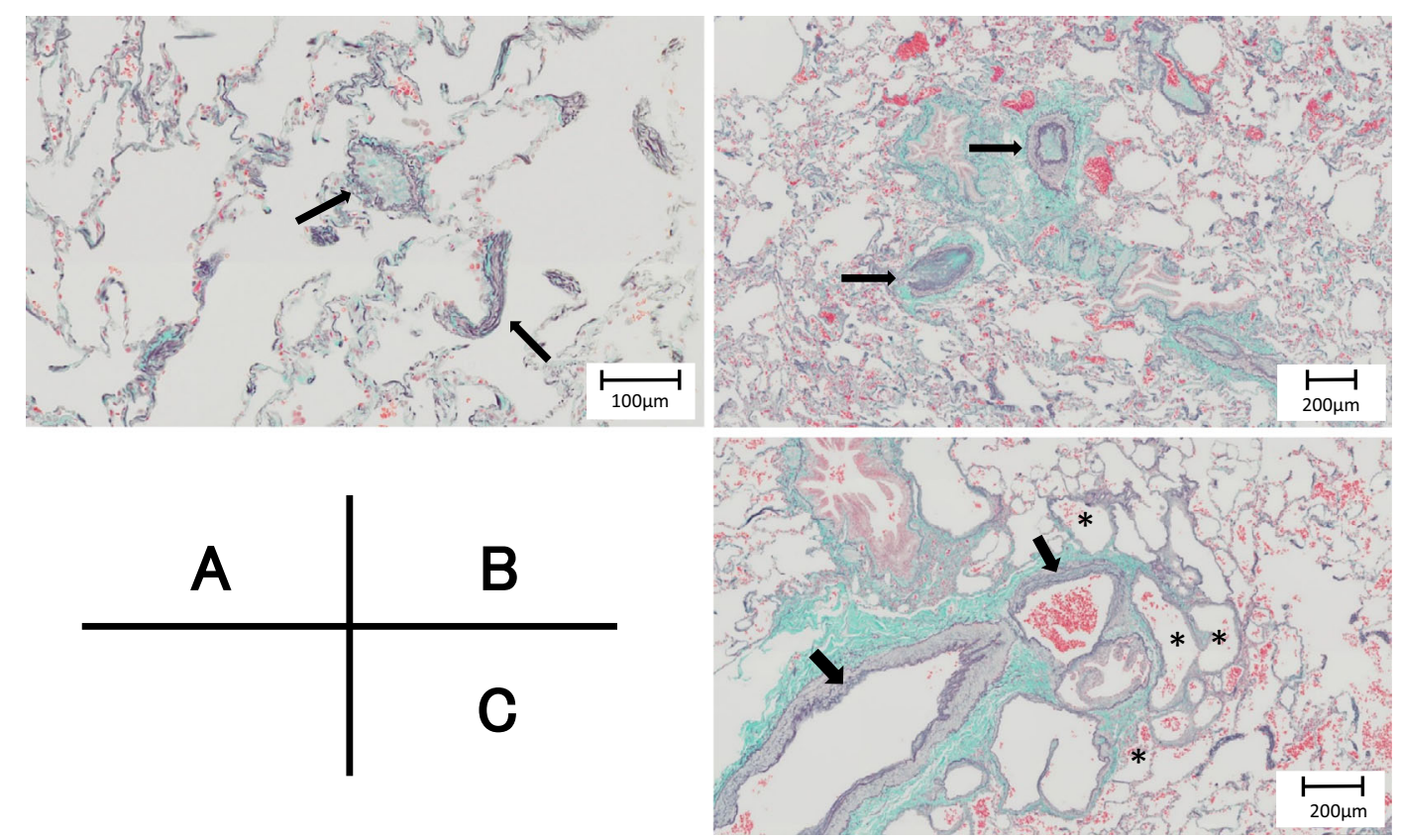

Figure 2. The histopathological findings of the lung tissue (Elastica-Masson Goldner staining). (A) Muscularization of the intra-acinar arterioles (arrows). (B) Muscular hypertrophy with intimal fibrotic proliferation in small pulmonary arteries (arrows). (C) The development of collateral channels (asterisks) around the occluded parent pulmonary artery (arrows), indicating the formation of plexiform lesions.

of age. Although the ASD was relatively large, there was no evidence to suggest a large left-to-right shunt, such as growth failure or exercise intolerance prior to the initial admission to the referral hospital. Thus, a risk factor other than the increased pulmonary blood flow might have been involved in the early development of severe PAH in our patient.

Genetic factors play a major role in the development of PAH (2). Both familial and idiopathic PAH are linked to mutations in the BMPR2 gene, a member of the transforming growth factor-beta (TGF- $\beta$ ) receptor superfamily. BMPR2 mutations are identified in approximately $70 \%$ of patients with familial PAH and $25 \%$ of patients with idiopathic PAH (2). The possible molecular mechanisms triggering PAH in such patients include abnormal proliferation of vascular smooth muscle cells and increased endothelial apoptosis in the pulmonary artery (5). Interestingly, BMPR2 mutations are also associated with patients with CHD and PAH. Roberts et al. reported that 6 out of 106 CHD patients with PAH had BMPR2 missense mutations (6). In all 6 cases, PAH was diagnosed before the patients reached their teens. However all of the patients had at least one posttricuspid shunt, such as VSD, atrio-ventricular septal defect, or patent ductus arteriosus, and the two of them had additional genetic syndrome. Thus, it is difficult to simply compare our patient to these cases. More recently, Liu et al. reported that the rate of $B M P R 2$ mutations in CHD patients with PVD was significantly higher in comparison to those without PVD (7.5\% vs. $1.2 \%, \mathrm{p}=0.004)$ (7). In contrast, Therrien et al. found no BMPR2 mutations in Eisenmenger syndrome patients with ASD or ASD controls (1), indicating that a $B M P R 2$ mutation is an important factor for triggering $\mathrm{PAH}$ in patients with CHD, whereas hemodynamic stress alone can produce irreversible PVD in patients with a large defect that causes Eisenmenger syndrome.

The BMPR2 gene is located on chromosome 2q33-q34 and has 13 exons (8). We identified a novel heterozygous missense mutation, c. $2474 \mathrm{~A}>\mathrm{G}$, in exon 12 of the BMPR2 gene. Exon 12 encodes an intracellular tail domain with an unknown function (8). To determine whether this mutation had been previously reported, we checked ExAC (http:// exac.broadinstitute.org/), ClinVar (https://www.ncbi.nlm.nih. gov/clinvar/) databases, and the Human Gene Mutation Database (HGMD, http://www.hgmd.org). However, it was not found in these databases and was considered to be novel. An in silico analysis, using SIFT (http://sift.jcvi.org) predicted that the c.2474A $>\mathrm{G}$ in $B M P R 2$ was damaging, while an analysis using Mutation Taster (http://www.mutationtaster. org/), predicted that it was disease causing, and an analysis using PolyPhen2 (http://genetics.bwh.harvard.edu/pph2/) predicted that it was probably damaging. This suggests that the mutation found in the present case occurred in a highly conserved site across species and that it may have affected the $B M P R 2$ function. The mode of inheritance was unclear because there were no available material from relatives and because the patient had no family history of PAH or CHD.

The early onset of irreversible PVD in the present case may be explained by the "two-hit" theory. Evans et al. reported that PAH patients with BMPR2 mutations presented at a younger age with more severe disease and had a poor 
prognosis in comparison to patients without BMPR2 mutations (9). Interactions between abnormal cell signals [via $B M P R 2$ mutation (5)] and hemodynamic overload (via ASD) can promote the rapid progression of pulmonary vascular lesions, resulting in severe PAH in patients in their teens. Thus, it seems useful for CHD patients with PAH to undergo a genetic analysis for idiopathic or familial PAH to evaluate the pathophysiology of developing PAH and in order to select an appropriate treatment. The determination of BMPR2 mutations appears to help in identifying CHD patients with PAH who have a high- risk of developing severe PVD at a much earlier stage in comparison to patients in whom it is expected based on the hemodynamic overload of the pulmonary circulation and the need for early corrective surgery. It may also be helpful to assess the risk of persistent or recurrent $\mathrm{PH}$ after the correction of the defect.

TGF- $\beta$ /BMP signaling is essential for heart development and the maintenance of the cardiac function; abnormalities in this signaling can cause congenital heart defects, such as atrioventricular defect and truncus arteriosus $(10,11)$. Talati et al. reported that both impaired fatty acid oxidation and the increased expression of the lipid transporter molecule CD36 are the mechanisms underlying the accumulation of lipids in the BMPR2 mutant RV (12). Van der Bruggen et al. showed that PAH patients with BMPR2 mutations had more severe RV dysfunction in comparison to those without the mutation, despite a similar RV afterload (13). Thus, the $B M P R 2$ mutation in our case may have affected both the progression of PVD and the abnormal atrial septal formation and/or the early development of RHF, which required lung transplantation.

\section{Conclusion}

In conclusion, we reported the case of a young man with a $B M P R 2$ gene mutation who presented with severe $\mathrm{PAH}$ in his early teens. A genetic analysis of CHD patients with PAH may help to evaluate the pathophysiological mechanisms underlying the development of PAH and facilitate the selection of an appropriate treatment.

The authors state that they have no Conflict of Interest (COI).

\section{Acknowledgement}

We would like to thank Miki Akiba, our transplant coordinator, for her outstanding dedication and commitment to the patient.

\section{References}

1. Therrien J, Rambihar S, Newman B, et al. Eisenmenger syndrome and atrial septal defect: nature or nurture? Can J Cardiol 22: 11331136, 2006.

2. Soubrier F, Chung WK, Machado R, et al. Genetics and genomics of pulmonary arterial hypertension. J Am Coll Cardiol 62: D13-D $21,2013$.

3. Diller GP, Gatzoulis MA. Pulmonary vascular disease in adults with congenital heart disease. Circulation 115: 1039-1050, 2007.

4. D'Alto M, Mahadevan VS. Pulmonary arterial hypertension associated with congenital heart disease. Eur Respir Rev 21: 328-337, 2012.

5. Rabinovitch M. Molecular pathogenesis of pulmonary arterial hypertension. J Clin Invest 122: 4306-4313, 2012.

6. Roberts KE, McElroy JJ, Wong WP, et al. BMPR2 mutations in pulmonary arterial hypertension with congenital heart disease. Eur Respir J 24: 371-374, 2004.

7. Liu D, Liu QQ, Guan LH, et al. BMPR2 mutation is a potential predisposing genetic risk factor for congenital heart disease associated pulmonary vascular disease. Int J Cardiol 211: 132-136, 2016.

8. Morse JH. Bone morphogenetic protein receptor 2 mutations in pulmonary hypertension. Chest 121: 50S-53S, 2002.

9. Evans JD, Girerd B, Montani D, et al. BMPR2 mutations and survival in pulmonary arterial hypertension: an individual participant data meta-analysis. Lancet Respir Med 4: 129-137, 2016.

10. Schneider MD, Gaussin V, Lyons KM. Tempting fate: BMP signals for cardiac morphogenesis. Cytokine Growth Factor Rev 14: 1-4, 2003.

11. Koitabashi N, Danner T, Zaiman AL, et al. Pivotal role of cardiomyocyte TGF-beta signaling in the murine pathological response to sustained pressure overload. J Clin Invest 121: 2301-2312, 2011.

12. Talati MH, Brittain EL, Fessel JP, et al. Mechanisms of lipid accumulation in the bone morphogenetic protein receptor type $2 \mathrm{mu}$ tant right ventricle. Am J Respir Crit Care Med 194: 719-728, 2016.

13. Van der Bruggen CE, Happe CM, Dorfmuller $\mathrm{P}$, et al. Bone morphogenetic protein receptor type 2 mutation in pulmonary arterial hypertension: A view on the right ventricle. Circulation 133: 1747$1760,2016$.

The Internal Medicine is an Open Access article distributed under the Creative Commons Attribution-NonCommercial-NoDerivatives 4.0 International License. To view the details of this license, please visit (https://creativecommons.org/licenses/ by-nc-nd/4.0/).

\footnotetext{
(C) 2017 The Japanese Society of Internal Medicine
} Intern Med 56: 3193-3197, 2017 